\title{
Beneficios y riesgos relacionados con el uso de la historia clínica electrónica
}

\author{
Advantages and risks associated with the use of electronic medical records
}

En el reciente Congreso del Centenario de la Sociedad Argentina de Pediatría, la Dra. Rainu Kaushal, Directora del Centro de Informática y Sistemas de Salud del Weill Cornell Medical College, al disertar sobre el uso de las historias clínicas electrónicas (HCE) describió las ventajas y desventajas asociadas con su empleo. Entre sus conclusiones, manifestó que el implementar las tecnologías de la información y de las comunicaciones (TIC) en el área de la salud no es tarea sencilla, ya que el uso de una HCE para la atención de los pacientes va más allá de poseer el hardware y el software, sino que obliga a cambios en la tarea asistencial que necesariamente deben ser tenidos en cuenta. Solo así, la implementación podrá ser exitosa y le brindará al pediatra las herramientas para una atención en salud de calidad y segura.

Por otro lado, la Organización Panamericana de la Salud publicó la "Estrategia y Plan de Acción sobre eSalud" para la región, con el objetivo de mejorar el acceso a los servicios de salud y su calidad con la ayuda de las TIC, la formación en alfabetización digital para generar sociedades más informadas y equitativas en el cuidado de la salud. ${ }^{1}$ Según la Organización Mundial de la Salud, la eSalud es "el apoyo que la utilización costo eficaz y segura de las TIC ofrece a la salud y a los ámbitos relacionados con ella, con inclusión de los servicios de atención de salud, la vigilancia y la documentación sanitarias, así como la educación, los conocimientos y las investigaciones en materia de salud". Dentro del ámbito de la eSalud se encuentra el uso de HCE, la telemedicina, la mSalud (salud con ayuda de dispositivos móviles) y el eLearning.

No existen estadísticas acerca del uso de la HCE en la Argentina, tanto en hospitales como en sanatorios y consultorios. Su empleo en los EE.UU. ha crecido en los últimos años (de 18,2\% en 2001 a 50,7\% en 2010), este aumento puede estar, en parte, relacionado con que el gobierno promulgó en 2009 la ley HITECH (Health Information Technology for Economic and Clinical Health - Tecnología de la Información para la Economía y Salud Clínica) que ofrece incentivos económicos a los profesionales de la salud que adopten el uso de HCE como parte de la reforma de salud. El otorgamiento del incentivo se evaluará en función de que los profesionales cuenten con una HCE certificada y aprobada por organismos designados y que la funcionalidad del registro electrónico pueda lograr mejoras significativas en la atención clínica y genere informes o reportes de salud para ser utilizados por el gobierno con fines epidemiológicos; esta última condición se conoce como "uso significativo" o "meaningful use". Estas condiciones han generado que las HCE sean el instrumento para medir la calidad en la atención y entre las propuestas a incluir se encuentran la completitud del calendario de vacunación acorde a la edad, evaluación del desarrollo madurativo junto con la medición antropométrica y nutricional con registro de índice de masa corporal, el control de la tensión arterial, el cuidado de los pacientes con asma y el cuidado de la faringitis, entre otras. ${ }^{2-4}$

La calidad de la atención en pediatría a nivel ambulatorio ha sido evaluada en un estudio que tomó 175 medidas de calidad sobre 12 áreas clínicas. Los investigadores revisaron los registros médicos de más de 1500 niños seleccionados al azar de 12 áreas metropolitanas de los Estados Unidos. El estudio encontró que menos del 50\% de los pacientes recibían la atención recomendada para la prevención y seguimiento. La calidad variaba mucho según el tipo de atención: los niños recibieron un $68 \%$ de la atención recomendada para problemas agudos, $53 \%$ de los cuidados de las enfermedades crónicas y $41 \%$ de la atención preventiva recomendada. ${ }^{5}$ A pesar de que no existen estudios similares en nuestro país, los estudios realizados sobre prescripciones del Programa Remediar encontraron que es variable el manejo de la bronquiolitis en comparación con lo recomendado por las guías de práctica clínica y existe una indicación o prescripción insuficiente de hierro para la prevención de la anemia en menores de dos años. Sobre la base de estos estudios, podemos afirmar que la calidad de la atención en nuestro país tampoco es óptima. ${ }^{6,7}$

El principal impulsor para el uso de las HCE es mejorar la calidad de la atención, ya que una HCE que ofrezca la posibilidad de registrar problemas de salud, evoluciones, resultados de estudios complementarios y prescripciones de fármacos puede contener alertas y recordatorios que brinden respaldo para la toma de decisiones 
en la actividad clínica. Es importante que una vez que se decida utilizar una HCE, ésta no se transforme en "silos de información" donde los datos no pueden ser compartidos; para ello, es necesario que se basen en estándares, como la Clasificación Internacional de Enfermedades, décima versión (CIE-10) que se utiliza para la codificación de patologías, y deben tener la posibilidad de ser interoperables, para poder comunicar información.

Sin embargo, una HCE puede no garantizar la calidad de la atención ni la seguridad de los pacientes, ya que aquellos programas que no han evaluado el impacto en el cuidado y los cambios asociados a la implementación pueden llevar a cometer "errores" que hasta ese momento no existían asociados a la nueva tecnología. Se ha descripto que estos nuevos errores pueden afectar la seguridad del paciente y se encuentran relacionados con diferentes factores, como: defectos del software y/o hardware que llevan a un mal funcionamiento durante el uso, el empleo inadecuado por parte del equipo de salud, la falta de interoperabilidad e interacción entre los sistemas de salud que resulta en la pérdida de datos o ingreso incorrecto de ellos, y una lenta transición en el paso del registro en papel al electrónico. Esto puede ocurrir cuando la implementación de los programas y aplicaciones no miden el impacto sobre los factores organizacionales y modificaciones del flujo de trabajo. . $^{8-11}$

Es importante evaluar el uso de las TIC en salud y la transformación sobre el cuidado para mejorar los resultados sobre el paciente, por lo que deben ser diseñados para brindar soporte a las necesidades de los profesionales de la salud y sus pacientes adaptados al entorno de cuidado. Para ello es necesario que los requerimientos de los usuarios y la complejidad del trabajo clínico sean analizados en primer lugar, seguido de una evaluación de todo el espectro de posibles soluciones, en lugar de examinar la gama actual de productos disponibles y la caracterización de las necesidades que puedan cumplir.
Es necesario contar con parámetros e indicadores adecuados para el éxito del uso de las HCE y éstos no deben ser solo sobre la adopción o el uso, sino más bien sobre el impacto en la salud de la población.

Dra. Paula Otero

Departamento de Pediatría, Departamento de Informática en Salud; Comité de Seguridad del Paciente. Escuela de Medicina, Hospital Italiano de Buenos Aires. paula.otero@hospitalitaliano.org.ar

doi:10.5546/aap.2011.476

1. Estrategia y Plan de Acción sobre eSalud de OPS/OMS. 2011 [Acceso: octubre 2011]. Disponible en: http:/ /www. paho.org/ict4health.

2. Zarlengo R. Meaningful use for the pediatrician. Med Health R I 2011;94(7):209-10.

3. Blumenthal D, Tavenner M. The "meaningful use" regulation for electronic health records. $N$ Engl J Med 2010;363(6):501-4.

4. Jensen RE, Chan KS, Weiner JP, Fowles JB, Neale SM. Implementing electronic health record-based quality measures for developmental screening. Pediatrics 2009;124(4):e648-54.

5. Mangione-Smith R, DeCristofaro AH,SetodjiCM,Keesey J, et al. The quality of ambulatory care delivered to children in the United States. N Engl J Med 2007;357(15):1515-23.

6. Bernztein R, Drake I. Subprescripción de hierro y variabilidad en el primer nivel de atención público de la Argentina. Arch Argent Pediatr 2008;106(4):320-7.

7. Bernztein R, Drake I, Elordi S. Variabilidad en el manejo de la bronquiolitis en el primer nivel de atención público de la Argentina. Arch Argent Pediatr 2008;106(3):205-11.

8. Sittig DF, Ash JS, Zhang J, Osheroff JA, Shabot MM. Lessons from "Unexpected increased mortality after implementation of a commercially sold computerized physician order entry system". Pediatrics 2006;118(2):797-801.

9. Sittig DF, Singh H. Eight rights of safe electronic health record use. JAMA 2009;302(10):1111-3.

10. Sittig DF, Singh H. Defining health information technology-related errors: new developments since to err is human. Arch Intern Med 2011;171(14):1281-4.

11. Koppel R, Metlay JP, Cohen A, Abaluck B, et al. Role of computerized physician order entry systems in facilitating medication errors. JAMA 2005;293(10):1197-203. 